Rabaska

Revue d'ethnologie de l'Amérique française

\title{
Chaire de recherche du Canada sur les migrations, les transferts et les communautés francophones (Université de Saint-Boniface)
}

\section{Yves Frenette et Yves Labrèche}

Volume 15, 2017

URI : https://id.erudit.org/iderudit/1041171ar

DOI : https://doi.org/10.7202/1041171ar

Aller au sommaire du numéro

Éditeur(s)

Société québécoise d'ethnologie

ISSN

1703-7433 (imprimé)

1916-7350 (numérique)

Découvrir la revue

Citer ce document

Frenette, Y. \& Labrèche, Y. (2017). Chaire de recherche du Canada sur les migrations, les transferts et les communautés francophones (Université de Saint-Boniface). Rabaska, 15, 333-335. https://doi.org/10.7202/1041171ar d'utilisation que vous pouvez consulter en ligne. 


\section{OUEST}

\section{Chaire de recherche du Canada sur les migrations, les transferts et les communautés francophones}

Université de Saint-Boniface 200, avenue de la Cathédrale Saint-Boniface (Manitoba) R2H 0H7
Téléphone : (204) 237-1818, poste 237

Courriel : yfrenette@ustboniface.ca Toile : ustboniface.ca/crc-mtcf

Le titulaire et les chercheurs associés à la CRC-MTCF ont poursuivi tout au cours de l'année 2016-2017 une foule d'activités qui visent à développer et promouvoir les études sur les phénomènes migratoires des francophones d'Amérique depuis l'établissement des premiers colons d'origine française au Nouveau Monde. L'éducation, la généalogie, la transmission du patrimoine culturel et linguistique et les processus interculturels font l'objet d'une attention particulière. En s'intéressant aussi aux migrations transnationales qui caractérisent les communautés contemporaines, les chercheurs visent à comprendre les enjeux identitaires des francophones en milieu minoritaire. Depuis la publication d'un premier rapport en 2014 (Frenette et Labrèche, Rabaska, vol. 12), plusieurs initiatives sur le plan de la recherche, du réseautage et de la diffusion ont permis de progresser dans l'atteinte des objectifs du programme. Pour l'année 2016-2017, ces activités ont été regroupées de la façon suivante : recherche historique, exploitation de bases de données, transferts culturels, migrations contemporaines et autres activités de mobilisation des connaissances ou de diffusion.

\section{Recherche historique}

Certaines activités de recherche et de mobilisation des connaissances touchent le continent nord-américain dans son ensemble : participation à titre de cochercheur dans des projets subventionnés de type Connexion (p. ex. " La recherche sur les francophonies d'Amérique : vers l'intégration interdisciplinaire et interrégionale ») ou encore la publication de synthèses (p. ex. "L'histoire sociale de l'Amérique française de 1763 à 1914 : état des lieux » dans les Cahiers Charlevoix en 2016, ou encore la participation à titre de président lors de colloques et de congrès ( $\mathrm{p}$. ex. séance « Migrations francophones en Amérique du Nord » lors du congrès de l'Institut d'histoire de l'Amérique française également en 2016).

Après quatre ans d'un labeur intensif, le titulaire, en collaboration avec les historiens Paul-André Linteau et Françoise Le Jeune, a fait paraître en avril 2017 aux Éditions du Boréal le livre Transposer la France. L'immigration française au Canada (1870-1914).

D'autres activités ont porté plus particulièrement sur le Manitoba et l'Ouest canadien (p. ex. recherches en vue d'une conférence sur « Les immigrants français dans la Prairie canadienne, 1870-1914 » prononcée à l'Université de Caen en mai 2016). Le titulaire de la CRC-MTCF, ainsi que son coordonnateur, ont présenté des données sur le peuplement et les archives orales du Manitoba français lors du colloque 
« Patrimoines documentaires communs et analyses des cultures en mouvement : Nouvelle Aquitaine, Amérique du Nord francophone » à l'Université de Poitiers en novembre 2016.

La Nouvelle-Angleterre a également fait l'objet d'une attention particulière cette année, notamment dans le cadre d'un vaste programme de développement de partenariat (voir ci-dessous, section Exploitation de bases de données). Ainsi, dès le mois de mai 2016, Y. Frenette présentait « La formation d'une petite-bourgeoisie canadienne-française en Nouvelle-Angleterre au XIX ${ }^{\mathrm{e}}$ siècle : une étude de cas » lors d'un colloque du Centre d'études nord-américaines, Université libre de Bruxelles. Par la suite, il publiait « L'historiographie des Franco-Américains de la NouvelleAngleterre, 1872-2015 » dans le Bulletin d'histoire politique, vol. 24, nº 2, hiver 2016, p. 75-103.

Enfin, d'autres publications et travaux en cours et issus de nombreuses collaborations ont eu comme objet le Québec et l'Ontario, et portaient entre autres sur la composition de la population ou encore les habitudes langagières des communautés francophones en milieu urbain (cas d'Ottawa et de Montréal).

\section{Exploitation de bases de données}

À la suite d'une première réunion du réseau de chercheurs et de partenaires communautaires du projet de Développement de partenariat Nouveaux regards sur l'occupation du continent nord-américain par la population canadienne-française, 1760-1914, des recherches se sont poursuivies au Québec et au Manitoba de même qu'en Nouvelle-Angleterre et dans le Centre-Ouest étatsunien (voir le site deploiements-francophones.ustboniface.ca).

Le titulaire de la CRC-MTCF qui est également directeur de ce projet participait par ailleurs à titre de membre du comité de pilotage d'un projet intitulé « Patrimoine oral et régional et dynamiques de l'interculturalité en contextes francophones », projet qui vise à constituer et à exploiter de grands corpus des deux côtés de l'Atlantique.

\section{Transferts culturels}

Des recherches sur l'histoire des manuels scolaires en usage au Manitoba français se sont poursuivies cette année afin de mieux articuler les données avec celles de l'Ontario et du Québec. De nouvelles analyses donneront lieu à une publication ainsi qu'à l'affinement de la base de données en vue de la rendre accessible sur Internet.

En 2016, Y. Frenette a également assuré la codirection de l'équipe francoquébécoise sur « Les récits de voyage et de migration comme modes de connaissance ethnographique : Canada, États-Unis, Europe ( $\mathrm{XIX}^{\mathrm{e}}-\mathrm{XX}^{\mathrm{e}}$ siècles) $)$. Les premiers récits seront bientôt mis en ligne sur l'Espace CIEQ de l'Université Laval et de l'Université du Québec à Trois-Rivières.

\section{Migrations contemporaines}

En octobre 2016, le titulaire ainsi que le coordonnateur de la CRC-MTCF ont participé à titre de membres du comité scientifique et présentateurs lors du colloque «Relations, pratiques et représentations de l'inclusion dans les communautés francophones en situation minoritaire : l'Ouest canadien au prisme des Amériques » qui avait lieu 
à l'Université de Régina. Un projet de publication découlant de ce colloque est en préparation. Ils ont également organisé une table ronde et un atelier dans le cadre du symposium " Migration, identités et refuge dans l'Ouest canadien », Institut canadien pour les identités et les migrations (IсIM) et Immigration Research West (IRW), à Winnipeg.

En outre, Y. Labrèche a travaillé à la codirection d'un numéro thématique de la revue Francophonies d'Amérique portant sur l'immigration francophone (à paraître). Il a également participé avec Y. Frenette et $\mathrm{M}$. Bamba à l'analyse de données ethnographiques récoltées tout récemment dans le cadre d'un projet intitulé « Arriver et partir : la francophonie de la Saskatchewan au début du XXI siècle ». La cueillette des données était financée par le CRFM (Centre canadien de recherche sur les francophonies minoritaires, Cité universitaire francophone, Université de Régina).

\section{Autres activités de mobilisation et de diffusion}

La CRC-MTCF a organisé la quatrième conférence annuelle « Robert-Painchaud » pour honorer la mémoire de l'historien d'origine manitobaine décédé prématurément en 1978. C'est l'auteur-compositeur et interprète Zachary Richard qui a prononcé en mars 2017 la conférence intitulée " L'histoire des Acadiens en Louisiane ».

Le titulaire a aussi contribué à diverses activités de mobilisation des connaissances se situant au-delà des axes proposés ci-dessus. Ainsi, en janvier et février 2017, il était professeur invité dans le cadre de l'International Research Training Group « Diversity : Mediating Difference in Transcultural Spaces », Universität Trier et Universität der Saarlandes.

À la suite de diverses contributions du titulaire et du coordonnateur de la CRCMтсF à l'analyse des témoignages et la publication des résultats des États généraux de la Société franco-manitobaine en 2016, Y. Labrèche participait en septembre 2016 à une table ronde intitulée : « La gouvernance communautaire dans l'Ouest canadien » dans le cadre du colloque « Engagement local, engagement global » du Centre d'études franco-canadiennes de l'Ouest qui se tenait à l'Université Simon Fraser (Vancouver).

Enfin, soulignons que le titulaire était intronisé dans l'Ordre des francophones d'Amérique en novembre 2016.

Yves Frenette et Yves Labrèche

\section{Institut pour le patrimoine de la francophonie de l'Ouest canadien}

Faculté Saint-Jean

Université d'Alberta

8406, rue Marie-Anne-Gaboury

Edmonton, Alberta T6C-4G9

Téléphone : (780) 465-8700

Télécopieur : (780) 465-8760

Courriel : saintjean@ualberta.ca

Toile : www.ualberta.ca/campus-saint-jean

Aperçu

L'année 2016-2017 a été la seconde année de direction de Claude Couture. Les faits 